\title{
Smoking reduces ovarian stromal blood flow in polycystic ovary syndrome patients
}

\author{
Özlen Emekçi Özay®i, Ali Cenk Özay® \\ Department of Obstetrics and Gynecology, Near East University, Nicosia, Cyprus
}

\begin{abstract}
Objectives: The objective of the current research is to compare hormonal, metabolic and ovarian stromal blood flow outcomes in smoking versus non-smoking polycystic ovary syndrome (PCOS) patients.

Material and methods: 79 PCOS women (Group 1) and 79 healthy subjects (Group 2) were recruited. Both groups were subdivided according to their smoking habits. Ovarian stromal blood flow was assessed with pulsatility and resistance index for both ovaries and compared among smoking women.

Results: The smokers in the PCOS group had statistically significant higher levels of pulsatility and resistance index in both ovaries compared to nonsmoker PCOS patients $(p<0.001)$. Comparison of ovarian stromal blood flow indexes shows that pulsatility and resistance index for both ovaries is statistically significantly high in smoking healthy patients compared to the nonsmoking group $(p<0.001)$. There is a positive correlation between smoking and free androgen index $(r=0.866$, $\mathrm{p}<0.001)$ for PCOS patients and healthy women.

Conclusions: Smoking reduces ovarian blood flow in PCOS patients and healthy subjects. Smoke components effect the vascular structure and form endothelial injuries that may reduce ovarian tissue perfusion. In this study, the positive correlation between smoking and free androgen index shows that the main effect of smoking on ovarian blood flow may be through androgen metabolism.
\end{abstract}

Key words: polycystic ovary syndrome; smoking; ovarian stromal doppler; pulsatility index; resistance index; cardiovascular risk

Ginekologia Polska 2020; 91, 4: 201-206

\section{INTRODUCTION}

Polycystic ovary syndrome (PCOS) was defined by Stein and Leventhal in 1935 and is characterized by hyperandrogenism, chronic anovulation and polycystic ovaries. It is a common endocrine disorder that affects 5-12\% of women worldwide [1, 2]. Signs and symptoms of PCOS include obesity, oligomenorrhea or amenorrhea, hirsutism and acne. PCOS can be diagnosed when two of the following three findings are present; anovulation, high levels of androgen and polycystic ovaries $[3,4]$. The syndrome can also form comorbid diseases such as impaired insulin sensitivity, type II diabetes, hyperlipidemia, and cardiac illness [3, 4]. It is recommended that PCOS patients are screened for metabolic syndrome and cardiovascular risk factors $[5,6]$. Every women with PCOS should get advices on how to adjust style of living to prevent metabolic complications and cardiovascular diseases.
The blood circulation of any organ is directly associated with its morphology and functions $[7,8]$. In women with PCOS, it has been shown that significant changes have occurred in vascularization of the ovarian stroma [7-10]. Battaglia et al. [7], Zaidi et al. [8], and Aleem et al. [9] established that, in cases with PCOS, important differences develop within the ovarian stroma circulation and they claimed that Doppler assays of ovarian stromal blood vessels may be useful for improving the investigation and could give more knowledge about the pathophysiology of the disease. In terms of systolic blood flow; there is a significant rise in the ovarian stromal velocities in women with PCOS compared to healthy women [7-11].

Clinical and experimental studies show that cigarette smoking alters the structures and functions of many organs and their blood circulation [12]. Based on the studies in the literature, the cigarette usage increases the incidence of car- 
diovascular diseases. Vasomotor dysfunction, inflammation and modifications of lipids, and the increase in androgen levels may be the reasons for the progression of vascular lesions and atherosclerosis $[13,14]$. There are studies in the literature showing the harmful effects of smoking on the vascular structures in the human body [12]. Kalfaoğlu et al. [15] assessed the cerebral blood flow by carotid and vertebral artery Doppler ultrasonography and showed that smoking reduces the blood flow to the brain. Cigarette usage impairs the functions and vascularization of the genital organs [16, 17]. Reproductive organs are effected by cigarette smoking, such as an earlier onset of menopause, higher infertility risk and lower fecundity rate $[16,17]$. Smoking is associated with endothelial dysfunction and an increase in arterial wall stiffness, and there is evidence showing the smoking effect on the genital organs based on the measurement of the blood flow of uterine artery, clitoral artery and labia minora arteries [17]. Nordenvall et. al. [18] and Albuquerque et al. [19] reported an increase in resistance index of the uterine artery in smoking women. In fact, no study has compared the effect of smoking on the ovarian doppler blood flow.

The harmful effects of smoking on health have been proven in non-PCOS women, which makes the possible effects of smoking on hormonal changes, especially androgens and ovarian blood flow changes in PCOS patients of interest [20]. The objective of the current study is to compare hormonal, metabolic and ovarian stromal blood flow outcomes in smoking versus non-smoking PCOS patients.

\section{MATERIAL AND METHODS}

The current prospective research was directed in the Division of Obstetrics and Gynecology at Near East University, Faculty of Medicine, Nicosia, Cyprus between February 2018 and June 2019. All participitants signed informed approval and local ethics committee accepted the study. All patients were young, nulliparous students from Near East University. Seventy-Nine PCOS women (Group 1) and 79 healthy subjects (Group 2) were recruited for the study. Both groups were evaluated according to their smoking habits. A total of 49 patients in the PCOS group and 39 women in the healthy group were smokers. Smoking was defined as 10 or more cigarettes per day. Medical and gynecologic patient history was asked from all the patients in the sample. The cases in group 2 had normal menstruation pattern and normal anatomical findings for baseline examination with transvaginal ultrasound. The diagnosis of PCOS was made according to Rotterdam Consensus criteria [4]. Ferriman Galwey (FGS) scoring system was used to define hirsutism and abnormal level of hirsutism was above 8. Exclusion aspects for the study were patient's age being more than 35 years, positive bhcg test result, high prolactin levels, thyroid disfunctions, congenital adrenal hyperplasia, any surgical procedure of the ovary in the past or medication/systemic illnesses that may affect the mechanics in the hypothalamus and pituatry gland, abnormal androgen secretion, diabetes, hormonal therapy and combined oral contraceptive usage six months prior the research. In addition, detection of ovarian cyst or follicle greater than ten milimeters or adnexial mass in a patients was accepted as elimination principle. Weight and height was measured by one clinician (Emekci Ozay $\mathrm{O}$ ) at the begining to calculate body mass index (BMI). Blood sample analysis were done to measure the prolactin ( $\mathrm{PRL})$, thyroid stimulating hormone (TSH), follicle stimulating hormone (FSH), luteinizing hormone (LH), estradiol (E2), fasting glucose, fasting insulin, dehydroepiandrosterone sulfate (DHEAS), total and free testosterone, and sex hormone binding globulin (SHBG). Homeostatic model of insulin resistance (HOMA-IR) was used to determine insulin sensitivity. If the level of HOMA-IR assessed was more than 2.5 , it was defined as high resistancy in insulin. Free androgen index was calculated by the formula 100x (Total testosterone/SHBG). During the initial five days of the menstruation, blood specimens were taken 8 hours afterwards abstaining. In women with no menstruation, after bhcg test analysis being negative, progesteron challenge test was performed with $5 \mathrm{mg}$ /day medroxyprogesterone acetate (TARLUSAL; Deva Holding A.S., Istanbul, Turkey). To check the ovarian blood flow, the doppler measurements were done during the third day of menstruation in the morning (08.00-11.00 am) by one operator (Ozay, A.C). The ultrasound machine used was Voluson 730Expert equipped with a 7-9 MHz transvaginal transducer. To avoid biases, clinician performing the examination was blinded to the clinical and hormonal findings of the patients. All women emptied their urinary bladders before the ultrasound examination and they rested for at least $15 \mathrm{~min}$ before being scanned to diminish the outer factors on blood circulation. For the assesment of the stromal blood flow of the ovaries, color waves in the stroma were desired at a greatest expanse from the exteriors of the ovary. Blood vessels placed near to the surface of a follicle were not evaluated. Three serial analysis were performed and the mean utility was calculated for each factor. The pulsatility index (PI) and resistance index (RI) were otomatically documented by the ultrasound. Results are represented as mean \pm standard deviation. Statistical Program for Social Sciences (SPSS) version 16 was used in the analysis. $p<0.05$ is accepted as statistically significant.

\section{RESULTS}

A total of 158 patients' results were analyzed. Group 1 was formed from 79 PCOS patients, where $62 \%(n=49)$ were smokers. Group 2 was formed from 79 healthy controls and cigarette usage in this group was $49.4 \%(n=39)$. The comparison of the demographic characteristics, hormonal, metabolic status and ovarian stromal doppler findings of 
PCOS and healthy group is shown in Table 1. The smokers in the PCOS group had statistically significant higher levels of pulsatility and resistance index in both ovaries compared to non-smoking PCOS patients. (Tab. 2). Comparison of ovarian stromal blood flow indexes showed that pulsatility and resistance index for both ovaries is statistically significantly high in healthy smoking patients compared to the non-smoking group. (Tab. 3). Table 4 shows a comparison of the ovarian doppler indices of PCOS and healthy women who smoke. No statistically significant change was detected in ovarian blood flow between these groups. There was a positive correlation between the number of cigarettes consumed per day and free androgen index $(r=0.866$, $p<0.001$ ) (Fig. 1). Also, there was a positive correlation between total testosterone $(r=0.448, p<0.001)$, free testosterone $(r=0.423, p<0.001)$ and cigarettes smoked per day. SHBG $(r=-0.454, p<0.001)$ showed a negative correlation with cigarettes smoked per day.

\section{DISCUSSION}

There are rising opinions that PCOS women can have an elevated risk of vascular disorders in comparison with healthy women of an identical weiht and age. The rate of reproductive age women who smoke is as high as $30 \%$ and smoking increases the incidence of cardiovascular diseases and peripheral vascular disease [21, 22]. The present study focuses on the association between smoking and diverse ovarian stromal blood flow and its relations with cardiovascular risks in PCOS patients and controls.

The results obtained in the current study confirm previous reports [21] of significantly higher BMI, LH/FSH ratio, circulating androgen values, insulin resistance and higher ovarian stromal blood flow in PCOS patients compared with healthy subjects. In 1995, Battaglia et al. showed that the existence of ovarian stromal vascularization with a decreased resistance index has great diagnostic worth for polycystic ovary syndrome [7]. Elevated levels of LH can be the cause for the marked up stromal vascularization by cytokine activation, catecholaminergic stimulation and neoangiogenesis [24]. Our study data supports that PCOS patients have lower right and left ovarian resistance index. The reduced $\mathrm{PI}$ and $\mathrm{RI}$ results are potential signals of the added on number and dilatation of ovarian stromal vessels in PCOS [25]. Also, Agrawal et al. [26] claimed that in PCOS, an elevated level of vascular endothelial growth factor can be responsible for heightened ovarian stromal blood flow [26]. Similar to our analysis, many other studies in the literature show the increased ovarian blood flow as a common factor in PCOS compared to healthy women with normal menstrual cycles $[7,8,11,24,27]$. There are studies that show that insulin and insulin-like growth factor induce the angiogenesis and vasodilatation in the ovary, although

Table 1. Comparison of hormonal, metabolic status and doppler findings of PCOS and healthy women

\begin{tabular}{|c|c|c|c|}
\hline & $P \cos (n=79)$ & Control $(n=79)$ & *p \\
\hline Age [years] & $21.76 \pm 2.10$ & $22.24 \pm 2.27$ & 0.168 \\
\hline BMI $\left[\mathrm{kg} / \mathrm{m}^{2}\right]$ & $23.91 \pm 4.55$ & $22.14 \pm 3.62$ & 0.008 \\
\hline Waist/Hip Ratio & $0.87 \pm 0.20$ & $0.68 \pm 0.09$ & $<0.001$ \\
\hline FGS & $11.97 \pm 4.58$ & $4.05 \pm 1.68$ & $<0.001$ \\
\hline LH/FSH & $1.60 \pm 0.72$ & $0.90 \pm 0.27$ & $<0.001$ \\
\hline Free T [pg/mL] & $1.98 \pm 0.67$ & $0.89 \pm 0.34$ & $<0.001$ \\
\hline Total T [nmol/L] & $1.66 \pm 0.54$ & $0.79 \pm 0.27$ & $<0.001$ \\
\hline FAI & $4.52 \pm 3.31$ & $0.98 \pm 0.46$ & $<0.001$ \\
\hline DHEAS [ug/dL] & $422.89 \pm 134.91$ & $207.59 \pm 71.27$ & $<0.001$ \\
\hline SHBG [nmol/L] & $54.02 \pm 29.62$ & $87.43 \pm 23.06$ & $<0.001$ \\
\hline Androstenedione [ng/L] & $130.48 \pm 31.45$ & $75.76 \pm 15.68$ & $<0.001$ \\
\hline HOMA-IR & $1.87 \pm 0.71$ & $1.16 \pm 0.43$ & $<0.001$ \\
\hline Mean BP [mmHg] & $87.80 \pm 8.02$ & $78.98 \pm 7.81$ & $<0.001$ \\
\hline LOPI & $0.74 \pm 0.23$ & $0.86 \pm 0.24$ & 0.001 \\
\hline LORI & $0.48 \pm 0.13$ & $0.54 \pm 0.08$ & 0.004 \\
\hline ROPI & $0.70 \pm 0.23$ & $0.83 \pm 0.18$ & $<0.001$ \\
\hline RORI & $0.50 \pm 0.14$ & $0.54 \pm 0.07$ & 0.026 \\
\hline
\end{tabular}

$\mathrm{BMI}$ — Body mass index; FGS — Ferriman Gallwey score; LH — Luteinizing hormone; FSH — Follicle stimulating hormone; Free T — Free Testosterone; Total T — Total Testosterone; FAI — Free Androgen Index; DHEAS — Dehydroepiandrosterone sulphate; HOMA-IR — Homeostatic model for assessment of insulin resistance; Mean BP — Mean Blood Pressure; LOPI — Left ovarian pulsatility index; LORI — Left ovarian resistance index; ROPI — Right ovarian pulsatility index; RORI — Right ovarian resistance index. ${ }^{*} \mathrm{p}<0.05$ is statistically significant; Independent samples $t$ test 
Table 2. Comparison of hormonal, metabolic status and doppler findings of smoking and non-smoking PCOS patients

\begin{tabular}{|l|l|l|l|}
\hline & Smokers $(\mathbf{n}=\mathbf{4 9})$ & Nonsmokers $(\mathbf{n}=\mathbf{3 0})$ & $\mathbf{p}$ \\
\hline Age [years] & $21.49 \pm 1.65$ & $22.20 \pm 2.64$ & 0.193 \\
\hline BMI $\left[\mathrm{kg} / \mathrm{m}^{2}\right]$ & $23.75 \pm 4.70$ & $24.17 \pm 4.35$ & 0.697 \\
\hline Waist/hip ratio & $0.86 \pm 0.22$ & $0.88 \pm 0.18$ & 0.788 \\
\hline LOPI & $0.87 \pm 0.20$ & $0.53 \pm 0.06$ & $<0.001$ \\
\hline LORI & $0.54 \pm 0.12$ & $0.40 \pm 0.11$ & $<0.001$ \\
\hline ROPI & $0.81 \pm 0.23$ & $0.52 \pm 0.08$ & $<0.001$ \\
\hline RORI & $0.56 \pm 0.11$ & $0.39 \pm 0.12$ & $<0.001$ \\
\hline LH/FSH & $1.51 \pm 0.66$ & $1.74 \pm 0.81$ & 0.162 \\
\hline Free T [pg/mL] & $2.03 \pm 0.63$ & $1.90 \pm 0.72$ & 0.405 \\
\hline Total T [nmol/L] & $1.70 \pm 0.51$ & $1.58 \pm 0.58$ & 0.316 \\
\hline FAI & $5.34 \pm 3.67$ & $3.18 \pm 2.05$ & 0.001 \\
\hline DHEAS [ug/dL] & $430.10 \pm 121.71$ & $411.11 \pm 155.57$ & 0.571 \\
\hline SHBG [nmol/L] & $48.61 \pm 30.63$ & $62.86 \pm 26.02$ & 0.031 \\
\hline Androstenedione [ng/dl] & $126.09 \pm 26.06$ & $137.64 \pm 38.09$ & 0.150 \\
\hline HOMA-IR & $1.80 \pm 0.68$ & $1.97 \pm 0.75$ & 0.310 \\
\hline Mean BP [mmHg] & $86.48 \pm 7.46$ & $89.94 \pm 8.56$ & 0.063 \\
\hline BMI Body & & & \\
\hline
\end{tabular}

$\mathrm{BMI}$ — Body mass index; FGS — Ferriman Gallwey score; LH — Luteinizing hormone; FSH — Follicle stimulating hormone; Free T — Free Testosterone; Total T — Total Testosterone; FAI — Free Androgen Index; DHEAS — Dehydroepiandrosterone sulphate; HOMA-IR — Homeostatic model for assessment of insulin resistance; Mean BP — Mean Blood Pressure; LOPI — Left ovarian pulsatility index; LORI — Left ovarian resistance index; ROPI — Right ovarian pulsatility index; RORI — Right ovarian resistance index; ${ }^{*} \mathrm{p}<0.05$ is statistically significant; Paired samples $t$ test

Table 3. Comparison of hormonal, metabolic status and doppler findings of smoking and non-smoking healthy patients

\begin{tabular}{|l|l|l|l|}
\hline & Smokers $(\mathbf{n}=\mathbf{3 9})$ & Nonsmokers $(\mathbf{n}=\mathbf{4 0})$ & $\mathbf{p}$ \\
\hline Age [years] & $22.62 \pm 2.60$ & $21.88 \pm 1.86$ & 0.151 \\
\hline BMI [kg/m²] & $22.08 \pm 4.32$ & $22.20 \pm 2.83$ & 0.876 \\
\hline Waist/hip ratio & $0.67 \pm 0.10$ & $0.69 \pm 0.08$ & 0.361 \\
\hline LOPI & $0.92 \pm 0.25$ & $0.81 \pm 0.22$ & 0.035 \\
\hline LORI & $0.57 \pm 0.08$ & $0.51 \pm 0.07$ & 0.002 \\
\hline ROPI & $0.89 \pm 0.19$ & $0.77 \pm 0.15$ & 0.002 \\
\hline RORI & $0.56 \pm 0.07$ & $0.51 \pm 0.06$ & 0.003 \\
\hline LH/FSH & $0.90 \pm 0.27$ & $0.89 \pm 0.27$ & 0.942 \\
\hline Free T [pg/mL] & $0.96 \pm 0.42$ & $0.84 \pm 0.21$ & 0.103 \\
\hline Total T [nmol/L] & $0.84 \pm 0.30$ & $0.74 \pm 0.22$ & 0.109 \\
\hline FAI & $1.10 \pm 0.54$ & $0.86 \pm 0.33$ & 0.022 \\
\hline DHEAS [ug/dL] & $208.38 \pm 69.73$ & $206.81 \pm 73.62$ & 0.922 \\
\hline SHBG [nmol/L] & $84.92 \pm 27.88$ & $89.89 \pm 17.15$ & 0.345 \\
\hline Androstenedione [ng/dL] & $75.35 \pm 14.88$ & $76.16 \pm 16.60$ & 0.821 \\
\hline HOMA-IR & $1.12 \pm 0.42$ & $1.20 \pm 0.44$ & 0.411 \\
\hline Mean BP [mmHg] & $79.37 \pm 8.06$ & $78.60 \pm 7.64$ & 0.664 \\
\hline
\end{tabular}

BMI — Body mass index; LH — Luteinizing hormone; FSH — Follicle stimulating hormone; Free T — Free Testosterone; Total T — Total Testosterone; FAI — Free Androgen Index; DHEAS - Dehydroepiandrosterone sulphate; HOMA-IR — Homeostatic model for assessment of insulin resistance; Mean BP — Mean Blood Pressure; LOPI — Left ovarian pulsatility index; LORI — Left ovarian resistance index; ROPI — Right ovarian pulsatility index; RORI — Right ovarian resistance index; ${ }^{*} p<0.05$ is statistically significant; Paired samples t test

the certain pathway has not yet been explained [28]. In our study we found that insulin resistance and HOMA-IR values are higher in the PCOS group compared to healthy controls.
When we evaluate the smoking effect on ovarian stromal blood flow in PCOS patients, we established that smoking increases the resistance and pulsatility index for right and left 
Table 4. Comparison of ovarian stromal doppler findings among smoking women

\begin{tabular}{|l|l|l|l|}
\hline & PCOS $(\mathbf{n}=49)$ & Control $(\mathbf{n}=\mathbf{3 9})$ & $\mathbf{p}$ \\
\hline LOPI & $0.87 \pm 0.20$ & $0.92 \pm 0.25$ & 0.259 \\
\hline LORI & $0.54 \pm 0.12$ & $0.57 \pm 0.08$ & 0.221 \\
\hline ROPI & $0.81 \pm 0.23$ & $0.89 \pm 0.19$ & 0.091 \\
\hline RORI & $0.56 \pm 0.11$ & $0.56 \pm 0.07$ & 0.905 \\
\hline
\end{tabular}

LOPI — Left ovarian pulsatility index; LORI — Left ovarian resistance index; ROPI — Right ovarian pulsatility index; RORI — Right ovarian resistance index; ${ }^{*} p<0.05$ is statistically significant; Paired samples t test

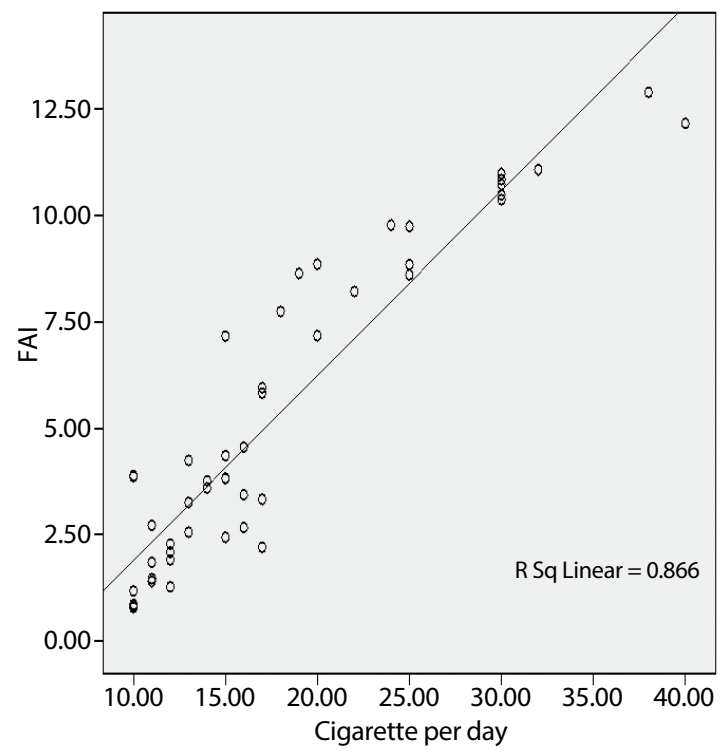

Figure 1. Correlation graphic of free androgen index and cigarettes smoked per day

ovaries in smoking PCOS compared to non-smoking PCOS patients. In addition, according to our study data, cigarette usage decreases the blood flow in healthy controls by increasing resistance and pulsatility index. Cigarette smoke causes blood vessels to increase stiffness, resistance, intima-media thickness, endothelial injury and decreases compliance and elasticity by increasing superoxide and endothelin-1 production [29]. Çifti et al. [30], studied the smoking effect on vascular structures and they found that smoking-related hemodynamic changes, endothelial dysfunction and increased stiffness were detected in subjects as young as 25 years of age. Battaglia et al. [17] showed that smoking reduces the blood flow in the genital organs in eumenorrheic women. For cases who use cigarrete for long time period, the vasoconstrictive outcomes of nicotine and the proatherogenic activity of different chemical components of smoke can cause a decreased perfusion in central and peripheral tissues [17].

Several studies have observed the effect of smoking in PCOS patients. This is the first research to observe the smoking effect on ovarian stromal blood flow in PCOS patients. Smoking may develop its effect of reducing the ovarian stromal blood flow in several ways. One possible route may be the increase in vascular stiffness, resistance and endothelial injury. Secondly, smoking may increase the androgen levels in blood circulation. Another influence of smoking could be its effect on insulin. However, in our study, we could not find any association between HOMA-IR values between smoking and nonsmoking PCOS patients. In fact, there was a significant increase in the free androgen index in smoking healthy women and PCOS patients. Also, there was a positive correlation between the number of cigarettes consumed per day and the free androgen index. In women with PCOS, effect of smoking on clinical, metabolic and endocrine outcomes were evaluated by Cupisti et al. [31]. It was found that PCOS cases who were smoking showed increased free testosterone and rising insulin resistance. In terms of acne, ovulatory function and hirsutism they declared no change between non-smokers and smokers [31]. Funabashi et al. [32] found that in women who smoke, nicotine does not change the levels of LH. [32] This finding is correlated with our research results and the LH/FSH ratio was not statistically significantly elevated in the smoking PCOS group compared to the non-smoker PCOS group in agreement with Cupisti el al. [31] The relation between androgens and nicotine can be associated with the metabolic route elaborated, as nicotine is mediated by cytochrome P450, which also plays a role in the biosynthesis of testosterone [33].

In our study, we also compared the smoking effect on ovarian stromal blood flow between PCOS and non-PCOS women. For both groups, ovarian stromal blood flow was reduced by smoking. The rate of decrease in the ovarian stromal blood flow in both groups was similar, and no statistically significant change was observed.

\section{CONCLUSIONS}

In conclusion, smoking reduces the ovarian stromal blood flow in PCOS and non-PCOS women. Nicotine has a vasoconstrictive effect and the endothelial injury of other smoke components may reduce ovarian tissue perfusion resulting in decreased ovarian stromal blood flow. In this study, the positive correlation between smoking and free 
androgen index shows that the main effect of smoking on ovarian blood flow may be through androgen metabolism.

\section{Acknowledgements}

The composers announce no financial contact with any company. All the cases in the study group gave consent for publication for the results from the study. These documents can be given to the review board by the Editor of this journal.

\section{REFERENCES}

1. Stein I, Leventhal M. Amenorrhoea associated with bilateral polycystic ovaries. Am J Obstet Gynecol. 1935; 29: 181-185.

2. Asunción $\mathrm{M}$, Calvo RM, San Millán JL, et al. A prospective study of the prevalence of the polycystic ovary syndrome in unselected Caucasian women from Spain. J Clin Endocrinol Metab. 2000; 85(7): 2434-2438, doi: 10.1210/jcem.85.7.6682, indexed in Pubmed: 10902790.

3. Azziz R, Carmina E, Dewailly D, et al. Androgen Excess Society. Positions statement: criteria for defining polycystic ovary syndrome as a predominantly hyperandrogenic syndrome: an Androgen Excess Society guideline. J Clin Endocrinol Metab. 2006; 91(11): 4237-4245, doi: 10.1210/jc.2006-0178, indexed in Pubmed: 16940456.

4. Rotterdam ESHRE/ASRM-Sponsored PCOS Consensus Workshop Group, Rotterdam ESHRE/ASRM-Sponsored PCOS consensus workshop group. Revised 2003 consensus on diagnostic criteria and long-term health risks related to polycystic ovary syndrome (PCOS). Hum Reprod. 2004; 19(1): 41-47, doi: 10.1093/humrep/deh098, indexed in Pubmed: 14688154.

5. Glintborg D, Andersen M. An update on the pathogenesis, inflammation, and metabolism in hirsutism and polycystic ovary syndrome. Gynecol Endocrinol. 2010; 26(4): 281-296, doi: 10.3109/09513590903247873, indexed in Pubmed: 20141388.

6. Wild RA, Carmina E, Diamanti-Kandarakis E, et al. Assessment of cardiovascular risk and prevention of cardiovascular disease in women with the polycystic ovary syndrome: a consensus statement by the Androgen Excess and Polycystic Ovary Syndrome (AE-PCOS) Society. J Clin Endocrinol Metab. 2010; 95(5): 2038-2049, doi: 10.1210/jc.20092724, indexed in Pubmed: 20375205.

7. Battaglia C, Artini PG, D'Ambrogio G, et al. The role of color Doppler imaging in the diagnosis of polycystic ovary syndrome. Am J Obstet Gynecol. 1995; 172(1 Pt 1): 108-113, doi: 10.1016/0002-9378(95)90094-2, indexed in Pubmed: 7847515.

8. Zaidi J, Campbell S, Pittrof R, et al. Ovarian stromal blood flow in women with polycystic ovaries--a possible new marker for diagnosis? Hum Reprod. 1995; 10(8): 1992-1996, doi: 10.1093/oxfordjournals.humrep. a136222, indexed in Pubmed: 8567828.

9. Aleem FA, Predanic M. Transvaginal color Doppler determination of the ovarian and uterine blood flow characteristics in polycystic ovary disease. Fertil Steril. 1996; 65(3): 510-516, doi: 10.1016/s0015-0282(16)58145-x, indexed in Pubmed: 8774278.

10. Bostanci MS, Sagsoz N, Noyan V. Comprasion of ovarian stromal and uterine artery blood flow measured by color Doppler ultrasonography in polycystic ovary syndrome patients and patients with ultrasonographic evidence of polycystic. J Clin Gynecol Obstet. 2013; 2: 20-26, doi: 10.4021/jcgo85w.

11. Pan $\mathrm{HA}, \mathrm{Wu} M \mathrm{MH}, \mathrm{Cheng} Y \mathrm{YC}$, et al. Quantification of Doppler signal in polycystic ovary syndrome using three-dimensional power Doppler ultrasonography: a possible new marker for diagnosis. Hum Reprod. 2002; 17(1): 201-206, doi: 10.1093/humrep/17.1.201, indexed in Pubmed: 11756388.

12. Rahman MM, Laher I. Structural and functional alteration of blood vessels caused by cigarette smoking: an overview of molecular mechanisms. Curr Vasc Pharmacol. 2007; 5(4): 276-292, doi: 10.2174/157016107782023406, indexed in Pubmed: 17979794.

13. Li J, Wu Q, Wu XK, et al. Effect of exposure to second-hand smoke from husbands on biochemical hyperandrogenism, metabolic syndrome and conception rates in women with polycystic ovary syndrome undergoing ovulation induction. Human Reproduction. 2018; 33(4): 617-625, doi: 10.1093/humrep/dey027.

14. Price JF, Mowbray PI, Lee AJ, et al. Relationship between smoking and cardiovascular risk factors in the development of peripheral arterial disease and coronary artery disease: Edinburgh Artery Study. Eur Heart J. 1999; 20(5): 344-353, doi: 10.1053/euhj.1998.1194, indexed in Pubmed: 10206381.

15. Kalfaoglu ME, Hizal M, Kiyan A, et al. The effects of chronic smoking on total cerebral blood volume measured by carotid and vertebral artery doppler ultrasonography. J Clin Ultrasound. 2017; 45(9): 561-565, doi: 10.1002/jcu.22513, indexed in Pubmed: 28656716.

16. Dechanet $C$, Brunet $C$, Anahory $T$, et al. Effects of cigarette smoking on reproduction. Hum Reprod Update. 2011;17(1): 76-95, doi: 10.1093/humupd/dmq033, indexed in Pubmed: 20685716.

17. Battaglia C, Battaglia B, Mancini F, et al. Cigarette smoking decreases the genital vascularization in young healthy, eumenorrheic women. J Sex Med. 2011; 8(6): 1717-1725, doi: 10.1111/j.1743-6109.2011.0225 7.x, indexed in Pubmed: 21477023.

18. Nordenvall M, Ullberg U, Laurin J, et al. Placental morphology in relation to umbilical artery blood velocity waveforms. Eur J Obstet Gynecol Reprod Biol. 1991; 40(3): 179-190, doi: 10.1016/0028-2243(91)90115-2, indexed in Pubmed: 1879593.

19. Albuquerque $C A$, Smith $K R$, Johnson $C$, et al. Influence of maternal tobacco smoking during pregnancy on uterine, umbilical and fetal cerebral artery blood flows. Early Hum Dev. 2004; 80(1): 31-42, doi: 10.1016/j. earlhumdev.2004.05.004, indexed in Pubmed: 15363837.

20. Ambrose JA, Barua RS. The pathophysiology of cigarette smoking and cardiovascular disease: an update. J Am Coll Cardiol. 2004; 43(10): 17311737, doi: 10.1016/j.jacc.2003.12.047, indexed in Pubmed: 15145091.

21. Morotti E, Battaglia B, Fabbri R, et al. Cigarette smoking and cardiovascular risk in young women with polycystic ovary syndrome. Int J Fertil Steril. 2014; 7(4): 301-312, indexed in Pubmed: 24520500.

22. Battaglia $C$, Mancini F, Fabbri $R$, et al. Vascular risk in young women with polycystic ovary and polycystic ovary syndrome. Obstet Gynecol. 2008; 111(2 Pt 1): 385-395, doi: 10.1097/01.AOG.0000296657.41236.10, indexed in Pubmed: 18238977.

23. Elmashad Al. Impact of laparoscopic ovarian drilling on anti-Müllerian hormone levels and ovarian stromal blood flow using three-dimensional power Doppler in women with anovulatory polycystic ovary syndrome. Fertil Steril. 2011; 95(7): 2342-6, 2346.e1, doi: 10.1016/j. fertnstert.2011.03.093, indexed in Pubmed: 21514928.

24. Ozdemir O, Sari ME, Kalkan D, et al. Comprasion of ovarian stroma blood flow measured by color Doppler ultrasonography in polycystic ovary syndrome patients and healthy women with ultrasonographic evidence of polycystic. Gynecol Endocrinol. 2015; 31(4): 322-326, doi: 10.3109/09513590.2014.995617, indexed in Pubmed: 25558942

25. Loverro G, Vicino $M$, Lorusso $F$, et al. Polycystic ovary syndrome: relationship between insulin sensitivity, sex hormone levels and ovarian stromal blood flow. Gynecol Endocrinol. 2001; 15(2): 142-149, doi: 10.1080/gye.15.2.142.149, indexed in Pubmed: 11379011.

26. Agrawal R, Conway G, Sladkevicius $P$, et al. Serum vascular endothelial growth factor and Doppler blood flow velocities in in vitro fertilization: relevance to ovarian hyperstimulation syndrome and polycystic ovaries. Fertil Steril. 1998; 70(4):651-658, doi: 10.1016/s00150282(98)00249-0, indexed in Pubmed: 9797093.

27. Lam PoM, Johnson IR, Raine-Fenning NJ. Three-dimensional ultrasound features of the polycystic ovary and the effect of different phenotypic expressions on these parameters. Hum Reprod. 2007; 22(12):3116-3123, doi: 10.1093/humrep/dem218, indexed in Pubmed: 17947376.

28. Morotti E, Giovanni Artini P, Persico N, et al. Metformin metabolic and vascular effects in overweight/moderately obese hyperinsulinemic PCOS patients treated with contraceptive vaginal ring: a pilot study. Gynecol Endocrinol. 2019 [Epub ahead of print]: 1-8, doi: 10.1080/09513590.2019.1613361, indexed in Pubmed: 31081406.

29. Rahman MM, Laher I. Structural and functional alteration of blood vessels caused by cigarette smoking: an overview of molecular mechanisms. Curr Vasc Pharmacol. 2007; 5(4): 276-292, doi: 10.2174/157016107782023406, indexed in Pubmed: 17979794.

30. Ciftçi $O$, Günday $M$, Calişkan $M$, et al. Light cigarette smoking and vascular function. Acta Cardiol. 2013; 68(3): 255-261, doi: 10.1080/ac.68.3.2983419, indexed in Pubmed: 23882870.

31. Cupisti $S$, Häberle L, Dittrich R, et al. Smoking is associated with increased free testosterone and fasting insulin levels in women with polycystic ovary syndrome, resulting in aggravated insulin resistance. Fertil Steril. 2010; 94(2): 673-677, doi: 10.1016/j.fertnstert.2009.03.062, indexed in Pubmed: 19394003.

32. Funabashi T, Sano A, Mitsushima D, et al. Nicotine inhibits pulsatile luteinizing hormone secretion in human males but not in human females, and tolerance to this nicotine effect is lost within one week of quitting smoking. J Clin Endocrinol Metab. 2005; 90(7): 3908-3913, doi: 10.1210/jc.2005-0041, indexed in Pubmed: 15870124

33. Enea C, Boisseau N, Diaz V, et al. Biological factors and the determination of androgens in female subjects. Steroids. 2008; 73(12): 1203-1216, doi: 10.1016/j.steroids.2008.06.009, indexed in Pubmed: 18640139. 